\title{
Lessons from studies of complex genetic disorders: identification of FOXL2 - a novel transcription factor on the wing to fertility
}

\author{
Dagmar Führer \\ III. Department of Medicine, University of Leipzig, Leipzig, Germany \\ (Correspondence should be addressed to D Führer; Email: fued@medizin.uni-leipzig.de)
}

Disturbance of the hypothalamo-pituitary-ovarian axis is present in approximately $10 \%$ of women in infertile couples. Thereby, two pathophysiological scenarios can be distinguished. First, hypogonadotropic hypogonadism due to processes directly or indirectly affecting the hypothalamus and/or the pituitary gland and secondly, hypergonadotropic hypogonadotropism, which results from unresponsiveness of the ovaries to gonadotropins. Primary ovarian malfunction can result from a wide spectrum of medical conditions including infectious, immunological, neoplastic, metabolic and vascular disorders, as well as radiation and chemotherapy. However, little is known so far about the genetic components responsible for ovarian failure (1). Inactivating mutations in the G-protein coupled folliclestimulating hormone (FSH) receptor have been identified as rare causes of primary hypogonadism (2) with ovaries in the affected women reportedly harboring normal follicles. In contrast, developmental ovarian failure is characterized by absence or reduction in the number of germ cells, resulting in either primary amenorrhea or premature ovarian failure (POF), i.e. onset of menopause before the age of 40 . While familial segregation of POF has long been recognized, dissection of molecular events involved is difficult because many 'mechanistic' pathophysiological aspects are still not fully understood and not all the genetic players have yet been identified (1).

As a general rule, one way to shed light on a complicated medical issue is to study conditions in which the trait of interest is inherited following a Mendelian pattern. Thus, a widely used technique to proceed from a specific phenotype to genotype analysis comprises systematic, chromosome-wide linkage analysis using inherited, highly polymorphic microsatellite markers to define candidate regions. These are usually several megabases in size and can be further narrowed by similar marker techniques (3). A complementary elegant procedure involves identification and mapping of specific chromosomal translocations in patients with a defined phenotype using fluorescent in situ hybridisation (FISH) analysis with the hypothesis that the gene disrupted by the translocation is associated with, if not responsible for, the observed phenotype. These strategies have been successfully applied in positional cloning of many 'disease genes', e.g. in Pendred's syndrome, multiple endocrine neoplasia type I and polyglandular endocrine syndrome type I.

One well-known genetic disorder associated with ovarian failure is the XO genotype of Turner's syndrome. In fact, X-chromosomal deletions and translocations have been reported in several cases of ovarian failure without Turner's syndrome, yet the identification of the involved X-chromosomal genes is still awaited (4). Another heritable disorder, which can be associated with ovarian failure, is blepharophimosisptosis-epicanthus inversus syndrome (BPES). The persistent study of this rare and complex condition by several teams of geneticists has recently led to the identification of a novel member of the forkhead winged helix protein family, FOXL2, as the likely underlying genetic cause and a putative new crucial player in ovarian development, and thus fertility (5).

BPES is an autosomal dominantly inherited disorder (MIM 110100), whereby the affected patients exhibit characteristic facial abnormalities, i.e. small palpebral fissures, ptosis and a skinfold running inward and upward from the lower lid. Two forms of BPES exist. In type I, infertility in the form of ovarian failure is an adjunct to the condition and is sex-limited, i.e. only females are affected. In type II, only facial abnormalities are present (6). BPES type II was first mapped to chromosome 3q22-q23 in 1995 followed by the observation that BPES I maps to the same locus, which suggested involvement of a common gene in both syndromes $(7,8)$. On the basis of three translocational breakpoints ( $(\mathrm{t} 3 ; 4),(\mathrm{t} 3 ; 7)$ and $(\mathrm{t} 3 ; 21))$ identified within the mapped region, several groups attempted to identify the culprit by positional cloning $(5,9-11)$. However, putative candidate genes involving two retinol-binding proteins and a beta-coatamer protein were either not found to be mutated or were not expressed in the relevant tissues of affected BPES individuals. In another study, breakpoint mapping failed to yield relevant candidate genes (11).

Crisponi et al. (5) started their gene hunting from a balanced translocation (t3;7) on chromosome 3 identified in a male BPES patient and mapped the breakpoint localization within $2 \mathrm{~kb}$ of genomic DNA by DNA hybridization with yeast artificial chromosomes (YACs) 
to identify a $1 \mathrm{Mb}$ YAC spanning the breakpoint in the first place, followed by hybridization with bacterial and P1 artificial chromosomes to narrow the translocation breakpoint. Prediction of two novel genes localized within the breakpoint region was made through several complementary methodological approaches involving computer assisted-analysis, DNA library hybridization and 5-RACE (rapid amplification of cDNA ends) PCR. One gene, termed C3orf5, was reported to be encoded by 20 exons and was found to be ubiquitously expressed as a $1.4 \mathrm{~kb}$ transcript with no obvious similarities to other previously identified genes. In contrast, the second gene was found to be a single exon gene with a $2.7 \mathrm{~kb}$ transcript encoding a protein of approximately 376 amino acids. The latter gene shared common features with members of the winged helix/forkhead protein family, and has been named FOXL2. Crisponi et al. (5) then succeeded in sequencing both novel genes in a number of BPES type I $(n=4)$ and BPES type II $(n=2)$ families, as well as a male patient with sporadic BPES, and were fortunate to identify genetic modifications in all affected BPES patients but not in the unaffected family members and a healthy control group $(n=100)$. Thereby all mutations were exclusively localized in the FOXL2 gene. Consistent with the clinical phenotype and the molecular genetic findings, mRNA expression studies of the FOXL2 mouse homologue Pfrk/FOXL2 gene demonstrated that the transcript is highly expressed during embryogenesis in the developing eyelids and is also expressed in mice ovary follicular cells. These findings suggest that a defective FOXL2 is the culprit in BPES types I and II. Further Northern Blot analysis of FOXL2 expression in human tissue revealed that the transcript is present in follicular cells surrounding the oocyte which underscores a potential role for FOXL2 in development and maintenance of the follicles.

The novel FOXL2 forkhead protein is the latest sibling in a group of, to date, six different forkhead domain proteins for which genetic defects have been identified in several complex human disorders, i.e. the thyroid transcription factor TTF-2 impaired in the thyroid agenesis, cleft palate and spiky hair syndrome (FOXE1; 9q22 (12)) and the genes implicated in the X-linked polyendocrinopathy, enteropathy and immune dysregulation syndrome (FOXP3; Xp11.23 (13, 14)), the hereditary lymphedema-distichiasis syndrome (FOXC2; 16q24.3 (15)), the anterior segment ocular dysgenesis and cataract syndrome (FOXE3; 1p32 (16)), as well as syndromic congenital glaucoma (FOXC1; 6p25 (17)). These proteins share a characteristic winged helix/fork head motif, i.e. a 100 amino acid DNA binding domain, which was first identified in Drosophila melanogaster and is constituted by two wings, three alpha helices and three beta sheets (for detailed topology see ref. 18). Interaction of the winged helix motif with the specific DNA region usually occurs via helix 3 and the respective DNA major grove, while wing 2 interacts with the DNA minor grove. Most of these interactions are mediated by polar amino acid residues through bridging water molecules and lead to conformational changes of the DNA resulting in specific gene transcription (18). The forkhead protein family currently comprises more than 100 genes identified in different organisms including 20 different human forkhead proteins $(18,19)$. They play a critical role during organogenesis and have also been implicated in the maintenance of cell differentiation and in tumorigenesis $(11,18,19)$.

The exciting findings by Crisponi et al. (5) have very recently been confirmed and extended by De Baere and co-workers (10), who have screened 33 probands from families with BPES type I $(n=3)$ and type II $(n=$ 9), 14 patients with sporadic occurrence of BPES, seven families with unknown BPES type (due to lack of affected females or pre-pubertal females), as well as 30 women with isolated POF. Besides the impressive number of heterozygous novel mutations $(n=17)$ identified, the study of De Baere et al. (10) provides important further insights into a pleiotropic function of the novel forkhead protein. In fact, similarly to Crisponi et al. (5), De Baere and co-workers (10) observed that the presence of either BPES type I or II crucially depends on the nature of the FOXL2 alteration (Fig. 1). Thus, a truncated FOXL2 protein is present in BPES type I. FOXL2 truncation can involve complete or incomplete loss of the forkhead domain by mutations upstream $(n=1)$ or within the forkhead domain $(n=4)$, or a protein truncation due to mutations further downstream $(n=7$, Fig. 1).

In addition, extension of FOXL2 is present in BPES type II. FOXL2 extension can result from in-frame duplication within the forkhead domain, e.g. by addition of five hydrophobic amino acids $(n=1)$, but more frequently appears to occur within the polyalanine region downstream of the forkhead domain $(n=4)$. Within this region with putative transcriptional repressive activity, addition of up to ten alanine residues has been described in BPES type II individuals. Moreover, three different insertion or deletion mutations further downstream of the polyalanine region have been identified to result in an extended FOXL2 protein. A similar pleiotropic effect has also been observed with mutations in other forkhead proteins, most notably FOXC1, which is implicated in several eye developmental defects (17).

Although De Baere et al. (10) reported FOXL2 mutations in $100 \%$ of their BPES type I families, 'only' $67 \%$ of BPES type II families, $71 \%$ of BPES families of unknown type and $57 \%$ of sporadic BPES patients were found to harbor FOXL2 mutations. Thus, involvement of other 'BPES genes' has been proposed with previous linkage analyses suggesting a second BPES candidate locus at $7 \mathrm{p} 21-\mathrm{p} 13$ (20). Alternatively, a position effect due to chromosomal rearrangement outside the coding region of the disease gene has been suggested. Position effects, believed to result in disruption of distant regulatory element and/or the minimal 


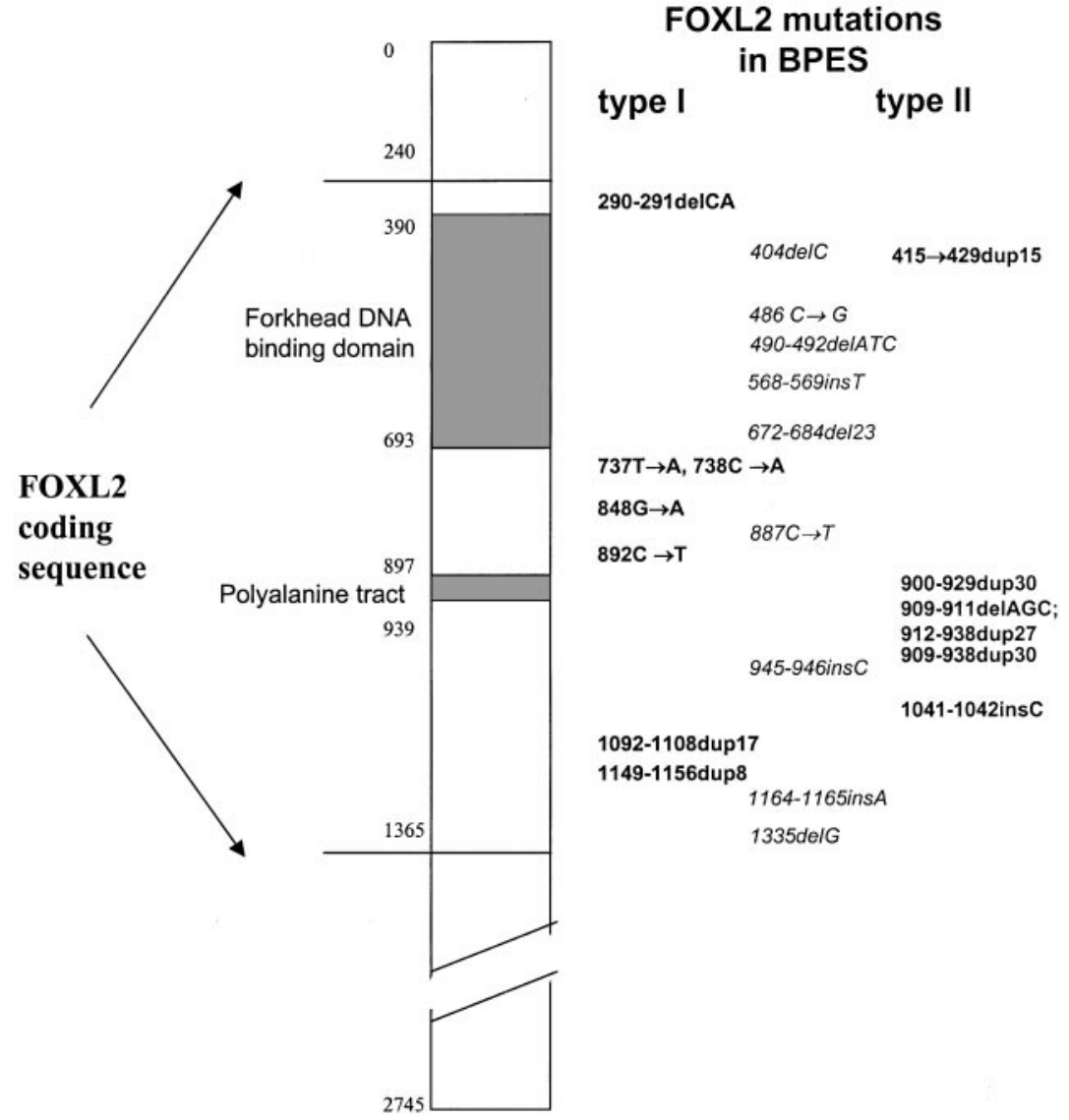

Figure 1 Diagram of the novel FOXL2 gene and mutations identified in patients with blepharophimosis-ptosis-epicanthus inversus syndrome (BPES) type I and II or patients with BPES of yet unknown type (italics). promoter, have been described in a number of rare human genetic disorders (21). Interestingly, absence of a FOXL2 mutation was also observed in the BPES patient with the balanced translocation (t3;7) from which Crisponi et al. (5) succeeded in cloning the BPES culprit.

Finally, no FOXL2 mutations were detected in a group of 30 women with non-syndromic POF. In view of the potentially high promise of FOXL2 for elucidating ovarian failure, this is the most disappointing finding of the De Baere study. However, as discussed above, a position effect and thus FOXL2 involvement in non-syndromic ovarian failure cannot (yet) be excluded.

Many other open questions remain to be answered with respect to FOXL2 transcriptional regulation, protein conformation, interaction partners and signaling pathways and ultimately protein function. Although highly speculative, an anti-apoptotic role of FOXL2 has been suggested because 'physiologic' atresia in ovarian follicles is notably enhanced in patients with POF (1). Moreover, a transforming growth factor- $\beta$ (TGF- $\beta$ ) signaling cascade has been proposed, since members of the TGF- $\beta$ family including the highly homologous forkhead transcription factor FAST-1 are implicated in ovarian development (22). In addition, the polled/intersex syndrome goat, which lacks horns and displays recessive XX sex reversal, has been proposed as a useful animal model for investigating FOXL2 function, after a candidate gene region homologous to the human FOXL2 locus has been identified in the goat model through linkage analysis (23).

Future detailed studies of functional FOXL2 impairment through the different mutations may contribute not only to clarify the highly variable morphological presentation of POF in BPES patients ranging from seemingly normal ovaries to streak gonades, but may also explain the interesting observation that female patients with likely BPES type II can exhibit some mild degree of fertility problems (10). This finding suggests a phenotypic overlap in the two forms of BPES. Furthermore, it is currently unclear how a heterozygous mutation in a putative transcriptional repressor can be sufficient for the BPES phenotype. Both a dominant negative mutational effect, or haplotype insufficiency, in which the truncated FOXL2 in BPES type I would represent a null allele and the extended FOXL2 protein in BPES type II a hypomorphic allele, have been hypothesized $(5,10)$.

With many open questions remaining to be answered after the identification of the sixth forkhead protein implicated in human disease, it will be exciting to discover more about the molecular and functional 
features of the pleiotropic FOXL2 and whether it is 'foxed' only in syndromic ovarian failure (5) or may indeed provide a 'wing' for novel insights into fertility and infertility.

\section{Acknowledgements}

I am grateful to Dr K Krohn, University of Leipzig for critical discussion of the manuscript.

\section{References}

1 Simpson JL \& Raikovic A. Ovarian differentiation and gonadal failure. American Journal Medical Genetics 199989 186-200.

2 Aittomaki K. Mutation in the follicle-stimulating hormone receptor gene causes hereditary hypergonadotropic ovarian failure. Cell 199582 959-968.

3 Schlotterer C. Evolutionary dynamics of microsatellite DNA. Chromosoma 2000109 65-71.

4 Prueitt RL, Ross JL \& Zinn AR. Physical mapping of nine Xq translocation breakpoints and identification of XPNPEP2 as a premature ovarian failure candidate gene. Cytogenetic Cellular Genetics $20008944-50$.

5 Crisponi L, Deiana M, Loi A, Chiappe F, Uda M, Amati P et al. The putative forkhead transcription factor FOXL2 is mutated in blepharophimosis/ptosis/epicanthus inversus syndrome. Nature Genetics 200127 159-166.

6 Zlotogora J, Sagi M \& Cohen T. The blepharophimosis, ptosis, and epicanthus inversus syndrome: delineation of two types. American Journal Human Genetics 19835 1020-1027.

7 Amati P, Chomel JC, Nivelon-Chevalier A, Gilgenkrantz S, Kitzig A \& Kaplan J. A gene for blepharophimosis-ptosis-epicanthus inversus syndrome maps to chromosome 3q23. Human Genetics 1995 $96213-215$.

8 Amati P, Gasparini P, Zlotogora J. Zelante L, Chomel JC, Kitzig A et al. A gene for premature ovarian failure associated with eyelid malformation maps to chromosome 3q-22-q23. American Journal Human Genetics 199658 1089-1092.

9 De Baere E, van Roy N, Speleman F, Kukushima Y, De Paepe A \& Messiaen C. Closing in on the BPES gene on 3q23: mapping of a de novo reciprocal translocation $\mathrm{t}(3 ; 4)$ (q2;p15.2) breakpoint within a $45 \mathrm{~kb}$ cosmid and mapping of three candidate genes, $\mathrm{RBP} 1, \mathrm{RBP} 2$, and $\mathrm{B}^{\prime}-\mathrm{COP}$, distal to the breakpoint. Genomics $19995770-78$.

10 De Baere E, Dixon MJ, Small KW, Jabs EW, Leroy BP, Devrienat K et al. Spectrum of FOXL2 gene mutations in blepharophimosisptosis-epicanthus inversus (BPES) families demonstrates a genotype-phenotype correlation. Human Molecular Genetics $2001101591-1600$.

11 Prueitt RL \& Zinn AR. A fork in the road to fertility. Nature Genetics $20012712-14$.

12 Clifton-Bligh RJ, Wentworth JM, Heinz P, Crisp MS, John R, Lazaruzs JH et al. Mutation of the gene encoding human TTF-2 associated with thyroid agenesis, cleft palate and choanal atresia. Nature Genetics 199819 399-401.

13 Bennet CL, Christie J, Ramsdell F, Brunkow ME, Ferguson PF \& Whitesell L. The immune dysregulation, polyendocrinopathy, enteropathy, X-linked syndrome (IPEX) is caused by mutations of FOXP3. Nature Genetics 200127 20-21.

14 Wildin RS, Ramsdell F, Peake J, Faravelli F, Casanova JL, Buist N et al. X-linked neonatal diabetes mellitus, enteropathy and endocrinopathy syndrome is the human equivalent of mouse scurfy. Nature Genetics 200127 18-20.

15 Fang J, Dagenais SL, Rickson RP, Arlt MF, Glynn MW, Gorski JL et al. Mutations in FOXC2 a forkhead family transcription factor are responsible for the hereditary lymphedema-distichiasis syndrome. American Journal Human Genetics 200067 182-188.

16 Semina EV, Brownell I, Mintz-Hittner HA, Murray JC \& Jamrich M. Mutations in the human forkhead transcription factor FOXE3 associated with anterior segment ocular dysgenesis and cataracts. Human Molecular Genetics 200110 21-26.

17 Nishimura DY, Searby CC \& Alward WL. A spectrum of FOXC1 mutations suggests gene dosage as a mechanism for developmental defects of the anterior chamber of the eye. American Journal Human Genetics 200168 64-72.

18 Gajiwala KS \& Burley SK. Winged helix proteins. Current Opinion in Structural Biology 200010 110-116.

19 Kaufmann E \& Knochel W. Five years on the wings of fork head. Mechanism of Development 199657 3-20.

20 Maw M, Kar B, Biswas J, Biswas P, Nancarrow D, Bridges R et al. Linkage of blepharophimosis syndrome in a large Indian pedigree to chromosome 7p. Human Molecular Genetics $1996 \mathbf{5}$ 2049-2054.

21 Kleinjan D \& van Heyningen V. Position effect in human genetic disease. Human Molecular Genetics 19987 1611-1618.

22 Chen X, Rubock MJ \& Whitman MA. A transcriptional partner for MAD proteins in TGF- $\beta$ signalling. Nature $19968691-696$.

23 Vaiman D, Schibler L, Oustry-Vaiman A, Pailhoux F, Goldammer T, Stevanovic M et al. High-resolution human/goat comparative map of the goat polled (intersex syndrome (PIS)): the human homologue contained in a human YAC from HSA3q23. Genomics 199956 1-9.

Received 14 September 2001

Accepted 24 September 2001 